\title{
SÍNTESIS Y CARACTERIZACIÓN DE HETEROPOLIOXOMETALATOS TIPO ANDERSON DE Ni-Mo(W) SOPORTADOS SOBRE ALÚMINA: ACTIVIDAD DE HIDRODESULFURACIÓN DE TIOFENO
}

\author{
SYNTHESIS AND CHARACTERIZATION OF \\ ALUMINA SUPPORTED Ni-Mo(W) ANDERSON \\ TYPE HETEROPOLYOXOANIONS: THIOPHENE \\ HYDRODESULFURIZATION ACTIVITY
}

\author{
Javier José Julio-Julio \\ Químico (c) \\ Universidad del Atlántico \\ Barranquilla, Colombia \\ javier_the4j@hotmail.com
}

\author{
Esneyder Puello-Polo \\ Ph. D. Ciencias \\ Universidad del Atlántico \\ Barranquilla, Colombia \\ esneyderpuello@mail.uniatlantico.edu.co
}

\author{
Joaquín Brito \\ Ph. D. Ciencias \\ Instituto Venezolano de Investigaciones Científicas \\ Estado Miranda, Venezuela \\ joabrito@ivic.gob.ve
}

\begin{abstract}
Resumen- Se sintetizaron heteropolioxometalatos tipo Anderson $\left(\mathrm{NH}_{4}\right)_{4}\left[\mathrm{NiMo}_{6-x} \mathrm{~W}_{\mathrm{x}} \mathbf{O}_{24} \mathrm{H}_{6}\right] \cdot 5 \mathrm{H}_{2} \mathrm{O}$ soportados sobre $\mathrm{y}-\mathrm{Al}_{3} \mathrm{O}_{2}$, utilizando el método de coprecipitación. Estos solidos fueron utilizados como precursores en la reacción de hidrodesulfuración (HDS) de tiofeno a $400^{\circ} \mathrm{C}$. Los precursores catalíticos se caracterizaron por propiedades texturales (área específica (B.E.T) y, distribución del diámetro y volumen de poros), difracción de rayos $X$ (DRX), espectroscopía infrarroja (FT-IR) y análisis elemental (CHON-S). Las áreas específicas estuvieron dentro del intervalo de 109 y $35 \mathrm{~m}^{2} / \mathrm{g}$ con una distribución del diámetro y volumen de poros de 8,9-9,6 nm y $0,11-0,34 \mathrm{~cm}^{3} / \mathrm{g}$, respectivamente. El análisis por DRX mostró la presencia de $\mathrm{NiWO}_{4},\left(\mathrm{NH}_{4}\right)_{4}\left[\mathrm{NiMo}_{6} \mathrm{O}_{24} \mathrm{H}_{6}\right] \cdot 5 \mathrm{H}_{2} \mathrm{O}$ y WO . Los análisis FT-IR determinaron la obtención de la estructura tipo Anderson, debido a las distintas flexiones y estiramientos de los grupos funcionales presentes. El precursor $\mathrm{NiMo}_{6}-\mathrm{O} / \mathrm{Al}$ fue el más activo en la HDS, con un porcentaje de conversión de $\mathbf{7 2 , 7 5 \%}$ e incluso fue más activo que el catalizador comercial AERO. Los resultados de HDS revelaron que la actividad catalítica en HDS se incrementa en el orden: $\mathrm{NiMo}_{6}-\mathrm{O} / \mathrm{Al}>$ AERO $>\mathrm{NiMo}_{3} \mathrm{~W}_{3}-\mathrm{O} / \mathrm{Al}>\mathrm{NiW}_{6}-0 / \mathrm{Al}$. La selectividad catalítica de precursores y el catalizador comercial (AERO) estuvo dirigida hacia cis-buteno.
\end{abstract}

Palabras clave- heteropolioxometalatos tipo Anderson; hidrodesulfuración; precursor oxídicos; NiMo(W).

\begin{abstract}
Alumina supported $\left(\mathrm{NH}_{4}\right)_{4}\left[\mathrm{NiMo}_{6}\right.$. $\left.{ }_{x} \mathrm{~W}_{\mathrm{x}} \mathrm{O}_{24} \mathrm{H}_{6}\right] \cdot 5 \mathrm{H}_{2} \mathrm{O}$ Anderson type heteropolyoxometalates were synthesized by coprecipitation. These solids were used as precursors in the thiophene hydrodesulfurization catalytic at $400{ }^{\circ} \mathrm{C}$. The catalytic precursors were characterized by textural properties (BET specific surface area and, total pore volume and pore diameter distribution), X-ray diffraction (XRD), IR spectroscopy (FTIR) and $S$ analysis (CHON-S). The specific surface area was between 109 and $35 \mathrm{~m}^{2} / \mathrm{g}$ with total pore volume and pore diameter distribution of $0,11-0,34 \mathrm{~cm}^{3} / \mathrm{g}$ and 8,9-9,6 $\mathrm{nm}$, respectively. XRD showed the presence of $\mathrm{NiWO}_{4},\left(\mathrm{NH}_{4}\right)_{4}\left[\mathrm{NiMo}_{6} \mathrm{O}_{24} \mathrm{H}_{6}\right] \cdot 5 \mathrm{H}_{2} \mathrm{O}$ and $\mathrm{WO}_{3}$. FTIR analysis can be assigned to the characteristic vibrations of Anderson-type structure, due to stretching and bending of the functional groups present. The $\mathrm{NiMo}_{6}-\mathrm{O} / \mathrm{Al}$ precursor was the most active in the HDS, it had a conversion of $72,75 \%$ and was even more active than the AERO commercial catalyst. The results of HDS revealed that the catalytic activity increases as follows: $\mathrm{NiMo}_{6}-\mathrm{O} / \mathrm{Al}>$ AERO $>\mathrm{NiMo}_{3} \mathrm{~W}_{3}-\mathrm{O} / \mathrm{Al}>\mathrm{NiW}_{6}-\mathrm{O} / \mathrm{Al}$. The catalytic selectivity of precursors and commercial catalyst (AERO) was directed towards the cis-butene.
\end{abstract}

Keywords- Anderson type heteropolyoxometalates; hydrodesulfurization, oxidic precursor, NiMo(W). 


\section{INTRODUCCIÓN}

El petróleo es la principal fuente de abastecimiento energético mundial, de este se obtienen gasolinas, querosén, combustibles pesados, diesel, lubricantes, asfaltos, parafinas, gas de refinería, coque y otros [1]. Esta fuente de energía es una mezcla compleja de hidrocarburos de bajo y alto peso molecular, conformados en mayor medida de carbono e hidrógeno en proporciones variables, además de pequeñas cantidades de algunos elementos, llamados heteroátomos como el oxígeno, azufre, nitrógeno y algunos metales como el V, $\mathrm{Ni}$, entre otros [2], [3]. El azufre en especial, constituye una de las impurezas más abundantes en las fracciones de petróleo, puesto que se encuentra inmerso en la estructura de una gran variedad de compuestos órgano-sulfurados, tales como tiofeno, benzotiofeno, dibenzotiofeno y aquil-familias de estos últimos [2]. Estos compuestos son, en general, de olor desagradable, altamente corrosivos, contaminantes atmosféricos y su presencia ha ocasionado un aumento en la contaminación del aire por la formación de $\mathrm{SO}_{x}$, además disminuye la calidad al diesel y la gasolina [4], [5], [6]. De allí, que en la búsqueda continua de mejorar la calidad del aire la agencia de protección del ambiente (EPA) y la directiva del parlamento europeo han establecido en los últimos años, que el contenido máximo permisible de azufre en los combustibles llegue a ser $\leq 10$ ppm y prevén llevar en algunos años las emisiones de azufre cercanas a cero. Por consiguiente, la industria petrolera ha tenido y tendrá como desafío reducir el contenido de azufre mejorando uno de los procesos catalíticos más importante a nivel mundial como lo es la hidrodesulfuración (HDS) [7], [8], [9]. Actualmente, se ha logrado con dificultad la meta de contenido de azufre utilizando catalizadores convencionales a base de sulfuros de $\mathrm{Ni}(\mathrm{Co})-\mathrm{Mo}(\mathrm{W}) / \mathrm{Al}_{2} \mathrm{O}_{3}$ con ciertas modificaciones al proceso de refinado, llevando en muchos casos al aumento en los costos de producción. Ante tal gravedad, el reto por cumplir es desarrollar catalizadores de HDS más activos y sin modificaciones al proceso de refinación; de aquí que recientemente diversos estudios hayan mostrado a los heteropolioxometalatos tipo Anderson $\left[\mathrm{XM}_{6} \mathrm{O}_{24} \mathrm{H}_{6}\right]^{\text {n- }}$ (donde $\mathrm{M}=\mathrm{Mo}^{\mathrm{Vl}} \mathrm{y} / \mathrm{o} \mathrm{W}^{\mathrm{Vl}}$ y X un metal de transición como $\mathrm{Ni}^{\prime \prime}$ o $\left.\mathrm{Co}^{1 / / 111}\right)$ como una alternativa para precursores catalíticos en los procesos de HDS [10], [12].
El desarrollo, caracterización y explotación de los heteropolioxometalatos tipo Anderson como catalizadores en HDS está en progreso y muchas investigaciones han evidenciado que estos compuestos en comparación con otros sistemas catalíticos (catalizadores convencionales) muestran grandes ventajas porque suprime pasos en la síntesis como calcinación, es decir, un solo paso de impregnación y secado, con el fin de preservar la estructura y evitar la formación de especies inactivas en HDS (aluminatos) [9], [13]; además, la presencia de heteroátomos en su estructura propicia propiedades redox y ácidas, y la posibilidad de existencia de efectos sinérgicos durante los procesos catalíticos; la solubilidad y conservación de la estructura en medio líquido aseguran una relación metálica bien definida [10], [11]. Por tal motivo, el propósito de este trabajo fue evaluar las propiedades catalíticas en HDS de tiofeno utilizando como precursor catalítico el heteropolioxomolibdato tipo Anderson $\left(\mathrm{NH}_{4}\right)_{4}\left[\mathrm{NiMo}_{6-x} \mathrm{~W}_{x} \mathrm{O}_{24} \mathrm{H}_{6}\right] \cdot 5 \mathrm{H}_{2} \mathrm{O}$ soportado sobre $\mathrm{y}-\mathrm{Al}_{3} \mathrm{O}_{2}$.

\section{PARTE EXPERIMENTAL}

\subsection{Síntesis del precursor catalítico Ni-Mo-W}

La fase oxídica Ni-Mo-W se obtuvo a partir de polioxomolibdatos tipo Anderson. La síntesis de las fases tipo Anderson $\left(\mathrm{NH}_{4}\right)_{4}\left[\mathrm{NiMo}_{6-x} \mathrm{~W}_{x} \mathrm{O}_{24} \mathrm{H}_{6}\right]$ - $5 \mathrm{H}_{2} \mathrm{O}(\mathrm{x}=0,3$ y 6$)$, se llevaron a cabo por coprecipitación de soluciones acuosas de heptamolibdato de amonio $\left(\left(\mathrm{NH}_{4}\right)_{6}\left[\mathrm{Mo}_{7} \mathrm{O}_{24}\right] \cdot 6 \mathrm{H}_{2} \mathrm{O}, \mathrm{T} \leq 60^{\circ} \mathrm{C}\right)$, paratungstato de amonio $\left(\left(\mathrm{NH}_{4}\right)_{6}\left[\mathrm{H}_{2} \mathrm{~W}_{12} \mathrm{O}_{40}\right] \bullet \mathrm{xH}_{2} \mathrm{O}\right)$ y nitrato de níquel $\left(\mathrm{Ni}\left(\mathrm{NO}_{3}\right)_{2} \bullet 6 \mathrm{H}_{2} \mathrm{O}\right)$ en proporciones estequiométricas que cumplieran con la relación $\mathrm{x}=0,3$ y 6 a temperatura ambiente y $\mathrm{pH}$ entre $5-6$, bajo agitación constante [14], [15]. Posteriormente, se filtró y lavó con agua destilada, para luego secarla a $105^{\circ} \mathrm{C}$ por $12 \mathrm{~h}$. Una vez obtenida la fase tipo Anderson, esta se soportó sobre alúmina $\left(\mathrm{y}-\mathrm{Al}_{2} \mathrm{O}_{3}\right)$ por impregnación en equilibrio a exceso de volumen de poros, tomando $3.0 \mathrm{~g}$ de $\mathrm{Y}^{-} \mathrm{Al}_{2} \mathrm{O}_{3}$ con $50 \mathrm{~mL}$ de solución $\left(\mathrm{NH}_{4}\right)_{4}\left[\mathrm{NiMo}_{6-x} \mathrm{~W}_{x} \mathrm{O}_{24} \mathrm{H}_{6}\right]$ - $5 \mathrm{H}_{2} \mathrm{O}(\mathrm{x}=0,3$ y 6$)$ a $50^{\circ} \mathrm{C}$ hasta sequedad del solvente y, por último, se secó a $105^{\circ} \mathrm{C}$ por $12 \mathrm{~h}$ [15].

Los precursores catalíticos se identificarán en este texto como $\mathrm{NiMo}_{6-\mathrm{x}} \mathrm{W}_{\mathrm{x}}-\mathrm{O} / \mathrm{Al}$, donde $\mathrm{x}=0,3$ y 6 indicará la relación metálica Mo/W. 


\subsection{Caracterización fisicoquímica de los precursores catalíticos Ni-Mo-W}

\subsubsection{Propiedades texturales $\left(A_{B E T}, V_{p}\right.$ y $\left.D_{p}\right)$}

El área específica de los precursores catalíticos fue determinada por el modelo de BrunaüerEmmett-Teller (BET) basado en la fisisorción de nitrógeno sobre la superficie de la muestra a la temperatura de nitrógeno líquido, mientras que los valores de distribución de volumen y tamaño de poros se obtuvo de la brazo de adsorción utilizando el método de Barrett, Joyner y Halenda (BJH). Las medidas se realizaron en un ASAP Micromeritics 2010 por adsorción de $\mathrm{N}_{2}$ a la temperatura de $77 \mathrm{~K}$.

\subsubsection{Espectroscopia infrarroja con transformada de Fourier (FT-IR)}

Con esta técnica se determinó de manera rápida los grupos funcionales que estaban presentes en los precursores catalíticos. Los análisis se realizaron con un espectrómetro marca Nicolet modelo iS10, empleando un intervalo de barrido entre $400-4000 \mathrm{~cm}^{-1}$.

\subsubsection{Difracción de rayos X (DRX) de polvos}

Esta técnica permitió identificar las distintas fases cristalinas de los precursores catalíticos. Se empleó, para ello, un difractómetro marca Siemens modelo D-5005 con radiación CuK $\alpha$ $(1,5456 \AA)$, donde se tomaron difractogramas dentro del intervalo de $2 \theta=10-90^{\circ}$, que fue donde aparecieron los picos de difracción de las fases en estudio.

\subsubsection{Análisis elemental CHON-S}

Este análisis se realizó con el fin de determinar el contenido total de carbono, hidrógeno, nitrógeno y azufre presente en los precursores catalíticos antes y después de reacción HDS. Los análisis se llevaron a cabo utilizando analizador elemental Fisons EA 1108.

\subsection{Medidas de la actividad catalítica de los precursores}

Se activaron los precursores catalíticos por presulfuración a presión atmosférica, colocando 250 mg del sólido en un reactor de flujo continuo y lecho fijo a $300^{\circ} \mathrm{C}$, pasándoles una mezcla de $100 \mathrm{~mL} / \mathrm{min}$ de $\mathrm{CS}_{2}(1 \mathrm{vol} \%) / / \mathrm{H}_{2}$ por 2 horas. Una vez activados, se midieron las actividades catalíticas de HDS, introduciendo al reactor a $400^{\circ} \mathrm{C} \mathrm{y}$ presión atmosférica una mezcla de $100 \mathrm{~mL} / \mathrm{min}$ de tiofeno $(3 \mathrm{vol} \%) / \mathrm{H}_{2}$. Las actividades catalíticas de HDS de tiofeno después de 3 h de reacción ( estado estacionario) se expresaron como moles de tiofeno convertidos a productos por gramos de catalizador por minutos ( $\mathrm{mol} \mathrm{Th} /$ gcat $\bullet \mathrm{min}$ ). El monitoreo de tiofeno y productos de reacción (butano, trans-buteno, 1-buteno, cis-buteno y 1,3-butadieno) durante el transcurso de reacción se realizó por cromatografía de gases en un cromatógrafo VARIAN-3700 con detector FID y una columna empacada Porapak a $170^{\circ} \mathrm{C}$ usando $\mathrm{N}_{2}$ como gas de arrastre [16].

\section{RESULTADOS}

\subsection{Propiedades texturales $\left(A_{B E T}, V_{p}\right.$ y $\left.D_{p}\right)$ de los precursores catalíticos}

En la Tabla I se presentan las áreas específicas de cada una de los materiales sintetizados, las cuales fueron determinadas por el método Brunaüer- Emmet Teller (BET), además de los valores de volumen de poros y diámetro de poros determinados por el método de Barrett-Joyner-Halenda (BJH). En esta se puede apreciar que el soporte catalítico $\left(\mathrm{Y}-\mathrm{Al}_{2} \mathrm{O}_{3}\right)$ presenta un área específica de $109 \mathrm{~m}^{2} / \mathrm{g}$, la cual disminuye progresivamente una vez que son impregnadas las fases tipo Anderson. Este comportamiento puede ser atribuido a la migración de parte de las fases tipo Anderson hacia el interior de los poros del soporte disminuyendo de esta manera su volumen y área específica [17], lo antes mencionado se soporta en el hecho de que el diámetro de poro en todos los precursores catalíticos no presenta diferencias significativas.

TABLA I

MEDIDAS TEXTURALES DE LOS PRECURSORES CATALÍTICOS: $\left(\mathrm{NH}_{4}\right)_{4}\left[\mathrm{NiMo}_{6-\mathrm{x}} \mathrm{W}_{\mathrm{x}} \mathrm{O}_{24} \mathrm{H}_{6}\right] \cdot 5 \mathrm{H}_{2} \mathrm{O}(\mathrm{x}=0 ; 3$ y 6$) / \mathrm{Y}-\mathrm{Al}_{2} \mathrm{O}_{3}$

\begin{tabular}{|c|c|c|c|}
\hline Material & $\begin{array}{c}\text { Área B.E.T } \\
\left(\mathrm{m}^{2} / \mathrm{g}\right)\end{array}$ & $V_{p}\left(\mathrm{~cm}^{3} / \mathrm{g}\right)$ & $\begin{array}{c}D_{p}-\mathrm{BJH} \\
(\mathrm{nm})\end{array}$ \\
\hline $\mathrm{Al}_{2} \mathrm{O}_{3}$ & 109 & 0,34 & 9,6 \\
\hline $\mathrm{NiMo}_{6}-\mathrm{O} / \mathrm{Al}$ & 61 & 0,18 & 8,9 \\
\hline $\mathrm{NiW}_{6}-\mathrm{O} / \mathrm{Al}$ & 55 & 0,14 & 8,9 \\
\hline $\mathrm{NiMo}_{3} \mathrm{~W}_{3}-\mathrm{O} / \mathrm{Al}$ & 35 & 0,11 & 9,3 \\
\hline
\end{tabular}

Fuente: autores. 
La Fig. 1 muestra que tanto la $\mathrm{y}-\mathrm{Al}_{2} \mathrm{O}_{3}$ como los precursores catalíticos presentaron isotermas del tipo IV con un ciclo histéresis tipo H2, característica de los materiales mesoporosos $\left(2 \mathrm{~nm}<\mathrm{D}_{\mathrm{p}}<\right.$ $50 \mathrm{~nm}$ ). Los sólidos obtenidos mantuvieron las características estructurales del soporte catalítico independientemente de la cantidad de fase tipo Anderson impregnada.

Fig. 1. ISOTERMAS DE ADSORCIÓN-DESORCIÓN DE N DE LOS PRECURSORES CATALÍTICOS SOPORTADOS SOBRE ALÚMINA:(») Y-AL $\mathrm{O}_{3} ;(\bullet)$ $\mathrm{NIMO}_{6}-\mathrm{O} / \mathrm{AL} ;$ ( $\Delta$ ) $\mathrm{NIW}_{6}-\mathrm{O} / \mathrm{AL} ;$ ( $(0) \mathrm{NIMO}_{3} \mathrm{~W}_{3}-\mathrm{O} / \mathrm{AL}$

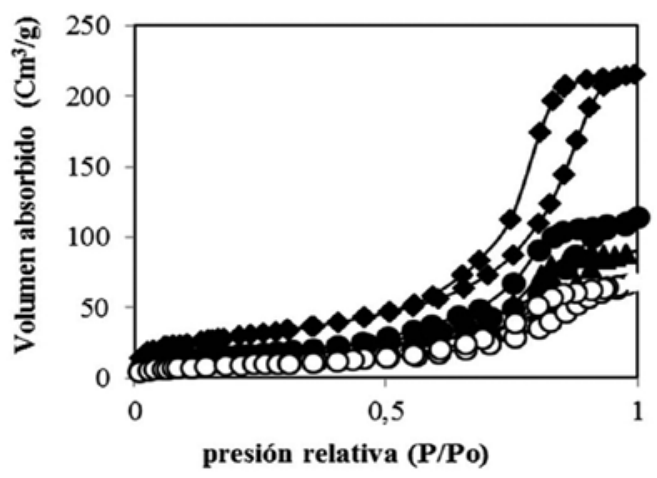

Fuente: autores.

En la Fig. 2 se aprecia la distribución de tamaño de poro de los precursores catalíticos, en donde hubo una disminución del tamaño de poro luego de la impregnación 3 a 7\% (no hay diferencia significativa). La cantidad de poros con los diámetros entre el rango de 8,9 y 9,6 disminuye conforme aumenta la cantidad metálica debido a la impregnación de las fases, corroborando lo que se mencionó anteriormente.

Fig. 2. DISTRIBUCIÓN DE TAMAÑO DE PORO DE LOS PRECURSORES CATALÍTICOS SOPORTADOS SOBRE ALÚMINA:(•) Y-AL $\mathrm{O}_{3} ;(\bullet) \mathrm{NIMO}_{6}-\mathrm{O} /$ $\mathrm{AL} ;(\triangle) \mathrm{NIW}_{6}-\mathrm{O} / \mathrm{AL} ;$ ( $(0) \mathrm{NIMO}_{3} \mathrm{~W}_{3}-\mathrm{O} / \mathrm{AL}$

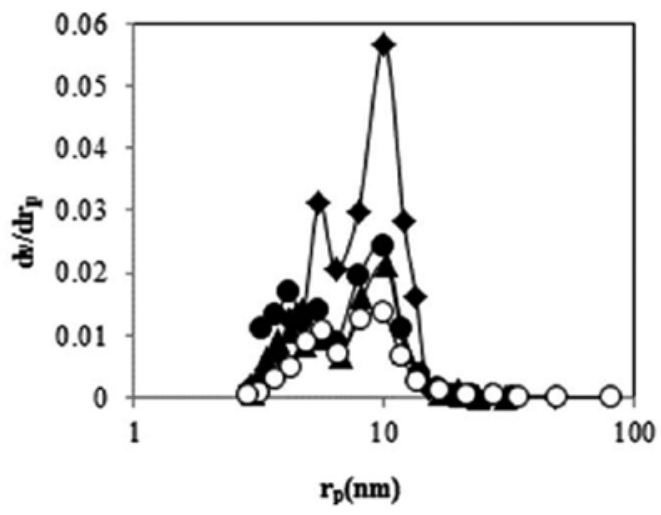

\subsection{Espectroscopia infrarrojo (FT-IR) de los precursores catalíticos}

El espectro vibracional de las fases tipo Anderson soportadas sobre alúmina se verificó a través de las características de los enlaces involucrados en esta estructura por FT-IR. Puesto que los heteropolioxometalatos tipo Anderson se caracterizan por la presencia de enlaces terminales $\mathrm{M}-\mathrm{O}_{\mathrm{t}}$ (oxígenos terminales) y enlaces puentes $\mathrm{M}-\mathrm{O}-\mathrm{M} / \mathrm{X}$ con átomos de oxígeno tipo $\mathrm{Oa}$ y $\mathrm{Ob}$, donde la (a) hace referencia a los átomos de oxígeno que se encuentran compartiendo vértices de octaedros $\mathrm{MO}_{6}$, y la (b) a átomos de oxígeno compartidos por tres poliedros (el octaedro central correspondiente al heteroátomo y dos octaedros del anillo de poliedros de molibdeno). El contra catión $\mathrm{NH}_{4}{ }^{+}$se encuentra coordinado a los oxígenos del heteropolioxoanión $\left(\left[\mathrm{NiMo}_{6-x} \mathrm{~W}_{\mathrm{x}} \mathrm{O}_{24} \mathrm{H}_{6}\right]^{4-}\right)$ y moléculas de agua [16]. En la figura 3 se presentan los espectros FT-IR de los precursores, en la figura 3-a se puede visualizar bandas alrededor de 3450 y $3184 \mathrm{~cm}^{-1}$ que se atribuyen a los estiramientos de los grupos $\mathrm{O}-\mathrm{H}$ de la alúmina; entre 1650 y $1410 \mathrm{~cm}^{-1}$ hay dos bandas que corresponden a vibraciones del grupo $\mathrm{O}-\mathrm{H}$ del agua adsorbida por el precursor. Las dos bandas de frecuencias más bajas $\left(780-470 \mathrm{~cm}^{-1}\right)$ son debidas a estiramientos y flexiones del enlace Al-O [18], [19]. Las Figs. 3-b, 3-c y 3-d presentan bandas entre 3424 y $3246 \mathrm{~cm}^{-1}$ atribuibles a estiramientos $\mathrm{N}-\mathrm{H}$ y $\mathrm{O}-\mathrm{H}$ del contra-catión y de las aguas de hidratación, respectivamente, además se puede observar a los $1628-1633 \mathrm{~cm}^{-1}$ las bandas de aguas de cristalización. La banda a 1400 $\mathrm{cm}^{-1}$ se debe a la flexión del grupo $\mathrm{NH}_{4}^{+}$. Las bandas en el rango de frecuencias de $950-850 \mathrm{~cm}^{-1}$ (3-b), son estiramientos simétricos y anti-simétricos del enlace terminal Mo- $\mathrm{O}_{\mathrm{t}} / \mathrm{W}-\mathrm{O}$, mientras que entre 750 y $560 \mathrm{~cm}^{-1}$ pertenecen a las vibraciones de las uniones puentes Mo/W-O-Mo/W (enlaces de longitud intermedia), lo cual nos indica la presencia de óxidos mixtos de Mo/W en este precursor. En la Fig. (3-d) aparecen bandas de enlaces terminales $\mathrm{W}-\mathrm{O}_{\mathrm{t}}$ y uniones puentes $\mathrm{W}-\mathrm{O}-\mathrm{W} / \mathrm{Ni}$. Es importante mencionar, que en la Fig. 3 (b-d) al soportar la fase Anderson sobre alúmina ocurre un leve corrimiento de las bandas hacia frecuencias menores si se compara con las del soporte catalítico, lo que permite suponer que hubo interacción entre la fase y la alúmina [20]. 
Fig. 3. ESPECTROS IR DE LOS PRECURSORES CATALÍTICOS SOPORTADOS SOBRE ALÚMINA:(A) $\Gamma-\mathrm{AL}_{2} \mathrm{O}_{3}$; (B) $\mathrm{NIMO}_{6}-\mathrm{O} / \mathrm{AL}$; (C) $\mathrm{NIW}_{6}-\mathrm{O} / \mathrm{AL}$; (D) $\mathrm{NIMO}_{3} \mathrm{~W}_{3}-\mathrm{O} / \mathrm{AL}$

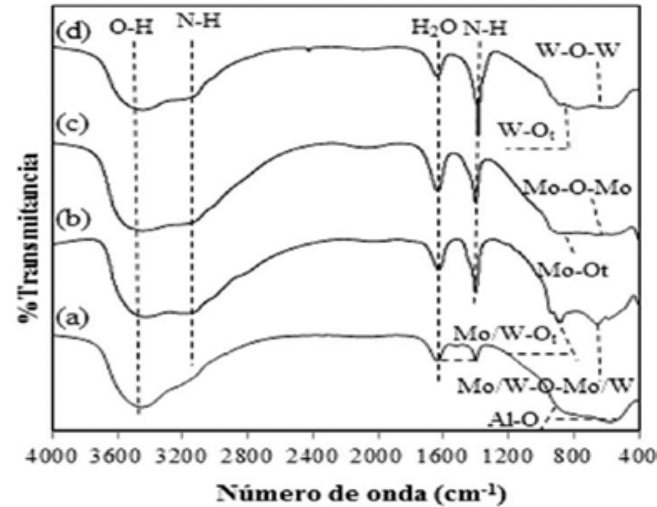

Fuente: autores.

\subsection{Difracción de rayos $X$ de los precursores catalíticos}

La asignación de las señales de difracción utilizó una base de datos de difracción [21]. En la Fig. 4-a se aprecia dos señales de difracción anchas de baja cristalinidad que sobresalen en $2 \theta=$ 45,90 y 67,10 características de la $\mathrm{Y}-\mathrm{Al}_{2} \mathrm{O}_{3}$ (JCPDF No 10-0425). Por otro lado, las Figs. 4-b y 4-c solo se muestran picos de difracción de $\mathrm{y}-\mathrm{Al}_{2} \mathrm{O}_{3}$ (JCPDF No 10-0425), lo cual permite suponer que los cristales de estas fases podrían ser demasiado pequeños, que no son observados por esta técnica analítica u ocurrió una buena dispersión de la fase activa sobre el soporte que no son detectables por DRX. La Fig. 7-d exhibe una mezcla de fases constituidas por $\mathrm{NiWO}_{4}$ (JCPDF No 015-0755) con $2 \theta=31,57 ; 36,60 ; 37,28 ; 39,15 ; 42,42 ; 49,09$; $\left(\mathrm{NH}_{4}\right)_{4}\left[\mathrm{NiMo}_{6} \mathrm{O}_{24} \mathrm{H}_{6}\right] \cdot 5 \mathrm{H}_{2} \mathrm{O}$ (JCPDF No 22-0506) $\operatorname{con} 2 \theta=32,08$ : 33,06; 34,09 y WO (JCPDF No 852460) con $2 \theta=23,22 ; 24,30 ; 28,14 ; 39,38 ; 55,63$. El hecho de que no hay una sola fase oxídica en esta muestra (Fig. 7-d), puede ser debido a que la fase tipo Anderson interaccionó con el soporte descomponiéndola o resultó inestable bajo las condiciones de impregnación [9], [22].

\subsection{Evaluación catalítica de HDS de tiofeno de los precursores catalíticos}

En la Fig. 5 se muestran los porcentajes de conversión de tiofeno de los precursores catalíticos en función del tiempo, datos que se aprecian en la Tabla II. El catalizador $\mathrm{NiMo}_{6}-\mathrm{O} / \mathrm{Al}$ presentó mayor actividad catalítica (73\%), posiblemente debido a mayor área superficial o mayor disposición de sitios activos en el catalizador (no ocurrió correlación entre la actividad y el área en todos los precursores). A pesar que no hay diferencia significativa en los \%p/p S para los precursores $\mathrm{NiW}_{6}-\mathrm{O} /$ Al y $\mathrm{NiMo}_{6}-\mathrm{O} / \mathrm{Al}$ una vez que son activados (Tabla III), sin embargo, el sólido $\mathrm{NiMo}_{6}-\mathrm{O} / \mathrm{Al}$ presentó la mayor actividad (no hubo correlación directa entre la actividad y el \% S), incluso que el $\mathrm{NiMo}_{3} \mathrm{~W}_{3}-\mathrm{O} / \mathrm{Al}$ que esperaba una mayor conversión del catalizador trimetálico $\mathrm{NiMo}_{3} \mathrm{~W}_{3}-\mathrm{O} / \mathrm{Al}$ como se encuentra en la literatura [23]; todo este comportamiento puede ser porque los catalizadores de tungsteno provenientes de sales oxídicas son difíciles de sulfurar, debido a que los enlaces W-O-Al y/o los W-O son más fuertes con respecto a los enlaces Mo-OAl y/o Mo-O [24], [25], [26].

Fig. 4. PATRONES DE DIFRACCIÓN DE RAYOS X DE LOS PRECURSORES CATALÍTICOS:(A) $\mathrm{r}-\mathrm{AL}_{2} \mathrm{O}_{3}$, (B) $\mathrm{NIMO}_{6}-\mathrm{O} / \mathrm{AL}$, (C) $\mathrm{NIW}_{6}-\mathrm{O} / \mathrm{AL}$, (D) $\mathrm{NIMO}_{3} \mathrm{~W}_{3}-\mathrm{O} /$

AL. (•) $\mathrm{AL}_{2} \mathrm{O}_{3},(\square)\left(\mathrm{NH}_{4}\right)_{4}\left[\mathrm{NIMO}_{6} \mathrm{O}_{24} \mathrm{H}_{6}\right] \cdot 5 \mathrm{H}_{2} \mathrm{O},(\square) \mathrm{NIWO}_{4} \mathrm{Y}(\Delta) \mathrm{WO}_{3}$

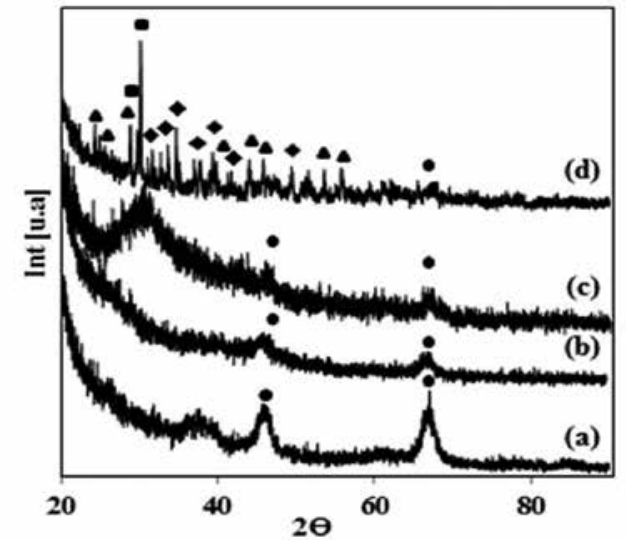

Fuente: autores.

Fig.5. \% CONVERSIÓN HDS DE TIOFENO DE PRECURSORES CATALÍTICOS: (•) $\mathrm{NIMO}_{6}-\mathrm{O} / \mathrm{AL} ;(\bullet) \mathrm{NIW}_{6}-\mathrm{O} / \mathrm{AL} ;(\bullet) \mathrm{NIMO}_{3} \mathrm{~W}_{3}-\mathrm{O} / \mathrm{AL}$

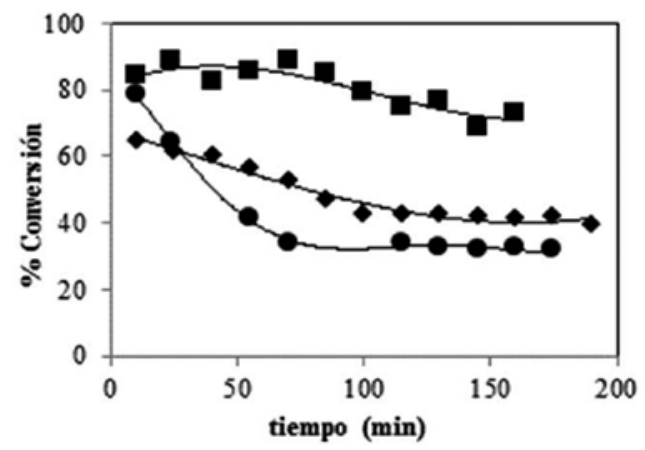

Fuente: autores. 
TABLA II

CONVERSIÓN (\%) Y ACTIVIDAD CATALÍTICA DE LOS PRECURSORES CATALITICOS EN LA REACCIÓN DE HDS DE TIOFENO

\begin{tabular}{|c|c|c|}
\hline Precursor catalítico & $\begin{array}{c}\text { Conversión } \\
\text { HDS }(\%)\end{array}$ & $\begin{array}{c}\text { Actividad catalítica } \times 10^{-4} \\
(\text { mol de Th conv/gCat } \bullet \text { min })\end{array}$ \\
\hline $\mathrm{NiMo}_{6}-\mathrm{O} / \mathrm{Al}$ & 73 & 3,02 \\
\hline $\mathrm{NiW}_{6}-\mathrm{O} / \mathrm{Al}$ & 32 & 1,31 \\
\hline $\mathrm{NiMo}_{3} \mathrm{~W}_{3}-\mathrm{O} / \mathrm{Al}$ & 40 & 1,64 \\
\hline Comercial (AERO) & 61 & 2,52 \\
\hline
\end{tabular}

TABLA III

PORCENTAJES MÁSICOS DE AZUFRE DE LOS PRECURSORES CATALÍTICOS ANTES Y DESPUÉS DE LA REACCIÓN DE HDS DE TIOFENO

\begin{tabular}{|l|c|c|}
\hline \multirow{2}{*}{ Precursor catalítico } & $\begin{array}{c}\text { Antes de reacción } \\
\text { HDS }\end{array}$ & $\begin{array}{c}\text { Después de } \\
\text { reacción HDS }\end{array}$ \\
\cline { 2 - 3 } & $\% p / p \mathrm{~S}$ & $\% p / p \mathrm{~S}$ \\
\hline $\mathrm{NiMo}_{6}-\mathrm{O} / \mathrm{Al}$ & 0,00 & 5,45 \\
\hline $\mathrm{NiW}_{6}-\mathrm{O} / \mathrm{Al}$ & 0,00 & 5,43 \\
\hline $\mathrm{NiMo}_{3} \mathrm{~W}_{3}-\mathrm{O} / \mathrm{Al}$ & 0,00 & 4,18 \\
\hline
\end{tabular}

En la Fig. 6 se muestran los resultados de selectividad en HDS de los precursores catalíticos en el estado estacionario ( $\sim 3$ horas). En general, se observa que todos los sólidos presentan una alta prioridad hacia cis-buteno (vía hidrogenólisis de tiofeno, poco poder hidrogenante del catalizador), este comportamiento catalítico se ve más marcado cuando el precursor contiene tungsteno, lo que permite sugerir que al estar menos reducidos los metales que constituyen el catalizador [27], este no dirige la reacción hacia la obtención de mayor cantidad de n-butano como se observa en $\mathrm{NiMo}_{6}-\mathrm{O} / \mathrm{Al}$.

Fig. 6. SELECTIVIDAD DE LA HDS DE TIOFENO PARA PRECURSORES CATALÍTICOS EN EL ESTADO ESTACIONARIO

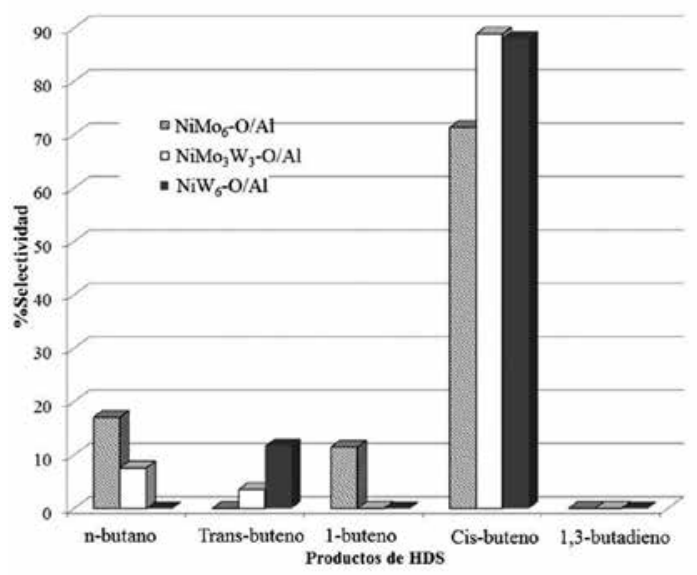

Fuente: autores.
En la Fig. 7 se hizo una comparación de las actividades catalíticas del precursor catalítico con mayor actividad y un precursor catalítico comercial (AERO, constituido de $\mathrm{NiMo} / \mathrm{Y}-\mathrm{Al}_{2} \mathrm{O}_{3}$ ), en la reacción de HDS de tiofeno. Se observa que el sólido $\mathrm{NiMo}_{6}-\mathrm{O} / \mathrm{Al}$ presentó mayor conversión inicial y después de $3 \mathrm{~h}$ de reacción HDS disminuye hasta hacerse algo estable, mientras que el precursor comercial exhibió una actividad constante durante la reacción HDS hasta los 150 minutos. Además, las selectividades de ambos precursores siguen la vía de formación de cis-buteno con mayor proporción para el AERO (Fig. 8). Es importante mencionar que a pesar de que las actividades son comparables, las relaciones metálicas de los catalizadores sintetizados en esta investigación son $\sim 0,14$ vs. $~ 0,33$ con que es preparado el catalizador convencional [9].

Fig. 7. COMPARACIÓN DE CONVERSIÓN (\%) HDS DE TIOFENO DE LOS PRECURSORES CATALÍTICOS. (๘) NIMO - -O/AL; ( $\bullet$ COMERCIAL (AERO)

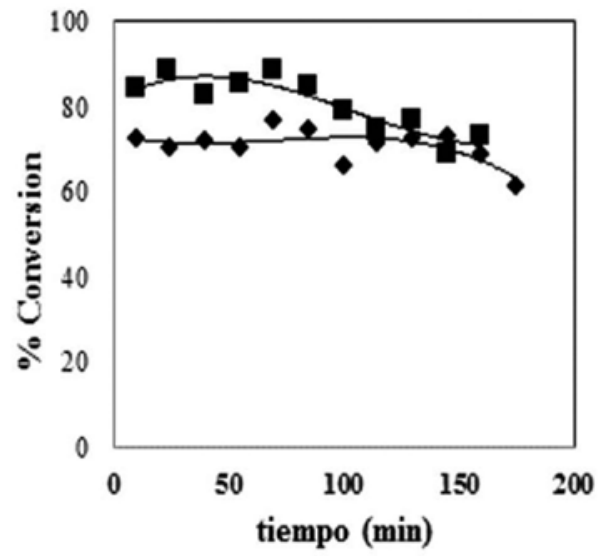

Fuente: autores.

Fig. 8. COMPARACIÓN DE SELECTIVIDAD (\%) DE LA HDS DE TIOFENO PARA PRECURSORES CATALÍTICOS (ロ) NIMO ${ }_{6}-\mathrm{O} / \mathrm{AL}$; $($ ) COMERCIAL (AERO)

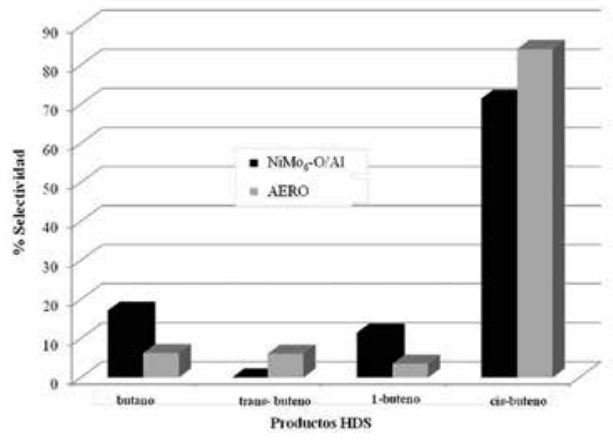

Fuente: autores. 


\section{CONCLUSIONES}

Todos los precursores mostraron isotermas tipo IV y ciclo de histéresis tipo $\mathrm{H} 2$, con áreas específicas entre 35-61 $\mathrm{m}^{2} / \mathrm{g}$, tamaño de poros entre 8,9-9,3 nm y volumen de poros entre 0,11$0,18 \mathrm{~cm}^{3} / \mathrm{g}$. Los análisis FT-IR de los precursores catalíticos mostraron señales Mo- $\mathrm{O}_{\mathrm{t}}$, W-O $\mathrm{O}_{\mathrm{t}}$, Mo-OMo, Mo-O-W, Ni-O-W y W-O-W correspondientes a las fases de óxidos mixtos de Ni-Mo-W. Los resultados de DRX presentaron fases cristalinas correspondiente a $\left(\mathrm{NH}_{4}\right)_{4}\left[\mathrm{NiMo}_{6} \mathrm{O}_{24} \mathrm{H}_{6}\right] \cdot 5 \mathrm{H}_{2} \mathrm{O}, \mathrm{Al}_{2} \mathrm{O}_{3}$, $\mathrm{WO}_{3}, \mathrm{NiWO}_{4}$. El precursor catalítico más activo en HDS fue $\mathrm{NiMo}_{6}-\mathrm{O} / \mathrm{Al}\left(\mathrm{NiMo}_{6}-\mathrm{O} / \mathrm{Al}(73 \%)>\right.$ AERO (61\%) $>\mathrm{NiMo}_{3} \mathrm{~W}_{3}-\mathrm{O} / \mathrm{Al}(40 \%)>\mathrm{NiW}_{6}-\mathrm{O} / \mathrm{Al}(32 \%)$ ), independientemente del \%p/p S encontrado en los sólidos después de reacción HDS. La selectividad catalítica de precursores y comercial (AERO) estuvo dirigida hacia la formación en mayor proporción de cis-buteno.

\section{AGRADECIMIENTOS}

Los autores desean expresar su agradecimiento al semillero de investigación en Oxi/Hidrotratamiento catalítico (UA), Laboratorio de Fisicoquímica de Superficies (Instituto Venezolano de Investigaciones Científicas (IVIC)) por la caracterización y reacciones catalíticas, a Yraida Díaz por toda la colaboración (IVIC), María Tosta (DRX), Liz Cubillaw (FTIR, IVIC) y E. Severino (CHNOS, IVIC) por el apoyo técnico, JJJJ dedica a Nellys Carolina Barrera Luna y Melanie Sofía.

\section{REFERENCIAS}

[1] C. Holanda, "Síntesis de catalizadores trimétalicos (MMo-W [M=Ni o Co]) Nanoestructurales soportados en óxido mixto Al-Ti," Tesis de titulación, Dpto. Ing. Química, Universidad Michoacana de San Nicolás de Hidalgo, Morelia Michoacán, 2010.

[2] Hernández, Z, "Caracterización química de crudos pesados en Venezuela," Trabajo de pregrado. Universidad Central de Venezuela, Caracas, 1976.

[3] J. Brito, "Estudio de Catalizadores Ni-Mo Carburados, Soportados Sobre TiO2-MCM-41 y su Actividad Catalítica en Reacciones de Hidrodesulfuración," Tesis de titulación, Dpto. Química, Universidad de Oriente, Cumaná-Venezuela, 2011.

[4] D. Salazar. y Vázquez, R, "Optimización de hidrotratamiento para la carga a desintegración catalítica fluidizada mediante simulación," Revista del Centro de Investigación, vol. 6, pp. 5-10, 2005.
[5] A. Salazar, "Caracterización de catalizadores de hidrotratamiento. Estudio de acidez,", Tesis M.Sc., Centro de Química, IVIC, 1982.

[6] S. Puello, "Carburos de Mo, Fe-Mo, Co-Mo y Ni-Mo soportados sobre carbón activado: actividades catalíticas de hidrodesulfuración y naturaleza del sitio activo," Tesis Ph. D, Instituto Venezolano de Investigaciones Científicas, Caracas, 2009.

[7] Luis, M.; Rives, A.; Hubaut, R.; Enbaid, B.; González, J. y Scott, C., "Study in surface science and catalyst," 1er. Taller Venezolano de Hidroprocesamiento de Crudos y Destilados, 1999.

[8] J.V. Babich, J.A. Moulijin, "Science and technology of novel processes for deep desulfurization of oil refinery stream". Fuel, no. 82, pp. 607-631, 2003.

[9] C. I. Cabello, I. L. Botto, H. J. Thomas, Appl. Catal. A: Gral., 197, p. 79, 2000.

[10] I. Pettiti, I. L. Botto, C. I. Cabello, S. Colonna, M. Faticanti, G. Minelli, P. Porta, H. J. Thomas, Appl. Catal. A: Gral., 220, p. 113, 2001.

[11] A. M. Maitra, N. W. Cant, Appl. Catal., 48, pp. 187-197, 1989.

[12] G.J. Siri, M.I. Morales, M.N. Blanco, H.J. Thomas, Appl. Catal, 19, p. 45, 1985.

[13] C. I. Cabello, I. L. Botto, H. J. Thomas, Thermochim. Acta, 232, p. 183, 1994.

[14] C. I. Cabello, M. Muñoz, E. Payen, H. J. Thomas, Catal. Lett., 92, pp. 69-73, 2004.

[15] Barbosa, A., "Caracterización fisicoquímica y ensayos catalíticos de los molibdatos de níquel, hierro y cobalto en la hidrodesulfuración de tiofeno," Trabajo de posgrado, M.Sc. Centro de Química, Instituto Venezolano de Investigaciones Científicas, Caracas, 1993.

[16] M. T. Pope, "Heteropoly and Isopoly Oxometalates", (Springer-Verlag), Berlin, New York, 1983, Cap. 1.

[17] Á. Sampieri, S. Pronier, S. Brunet, X. Carrier, C. Louis, J. Blanchard, K. Fajerwerg, M. Breysse, Microporous and Mesoporous Materials no. 130, pp. 130-141, 2010.

[18] Parry D.B., Samant, M.G., Seki, H., Philpott, M.R. y Ashley, K., Langmuir no. 9, pp. 1878-1887, 1993.

[19] Yang, H., Liu, M. y Ouyang, J., Applied Clay Science no. 47, pp. 438-443, 2010.

[20] M. T. Pope, A. Müller, Angew. Chem. Int. Ed. Engl. no. 30, pp. 34-48, 1991.

[21] Power Diffraction File, ICDD. Newtown Square, Philadelphia, 1995. 
[22] D.S. Kim, I.E. Wachs, K. Segawa, J. Catal. no. 149, pp. 268-277, 1994.

[23] Eijsboust, S., van Houter, F., Soled, K., Miseo, S., Anderson, G. and Fujita, K, “"NEBULA”: A Hydroprocessing catalyst with breakthrough activit," Science and Technology in Catalysis no. 145, pp. 407-410, 2003.

[24] Magnus, P.J., Schefer, B. y Moulijn, J.A., Amer. Chem. Soc. Petro. Div. Prep. no. 32, p. 329, 1987.
[25] Kabe, T., Qian, W., Funato, A., Okoshi, Y. y Ishihara, A., Phys. Chem. Chem. Phys. vol. 1, pp. 921-927, 1999.

[26] Thomas, R., Van Oers, M., de Beer, V.H., Mederna, J. y Moulinn, J.A., J. Catal. no. 76, pp. 241-253, 1982.

[27] PT.A. Zepeda, A. Infantes-Molina, J.N. Díaz de León, S. Fuentes, G. Alonso-Núnez, G. Torres-Otañez, B. Pawelec. Appl. Catal. A: Gen, no. 484, pp. 108-121, 2014. 